\title{
Efficacy of two soft-bristle toothbrushes in plaque removal. A randomized controlled trial
}

\author{
Gerson P. J. Langa', Francisco W. M. Gomes Muniz², Harry J. Rivera Oballe', Juliano \\ Cavagni', Stephanie Anagnostopoulos Friedrich', Zilson Malheiros ${ }^{3}$, Bernal Stewart ${ }^{3}$, \\ Cassiano Kuchenbecker Rösing?
}

\author{
1. Universidade Federal do Rio Grande do Sul, Faculdade de Odontologia, Departamento de Periodontia, Porto Alegre, \\ Brasil \\ 2. Universidade Federal de Pelotas, Faculdade de Odontologia, Departamento de Periodontia, Pelotas, Brasil \\ 3. Colgate Palmolive, Centro de Tecnologia Global, New Jersey, Estados Unidos
}

\begin{abstract}
The aim of the present examiner-blind randomized controlled clinical study was to compare the efficacy two soft-bristle toothbrushes in terms of plaque removal. Seventy volunteers were randomly allocated to Group A (tapered-tip toothbrush) or Group B (end-rounded toothbrush). At baseline appointment (Day 0), volunteers underwent plaque examination using the Improved Plaque Identification Index. Under supervision, they brushed their teeth for 1 minute with their assigned toothbrushes and the plaque examination was repeated. Volunteers continued the oral hygiene regimen (assigned toothbrush and a regular dentifrice provided by the researchers) for 7 days. The experimental procedures of Day 0 were then repeated. Separate statistical analyses were performed for mean percent reduction of plaque in the whole-mouth, interproximal and gumline scores at both times, using Mann-Whitney test, $p<0.05$. After a single
\end{abstract}

toothbrushing, on Day 0, mean percent plaque was significantly reduced in both groups $(p<0.05)$, with statistically greater

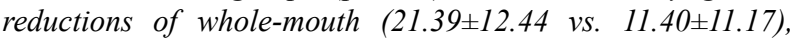
gumline (6.32 \pm 7.37 vs. 2.89 \pm 4.57$)$ and interproximal

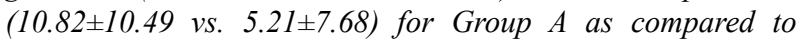
Group B. However, on day 7, no significant difference was observed between groups for whole-mouth (29.94 \pm 20.91 vs. $26.58 \pm 18.64)$, gumline (14.04 18.82 vs. $13.78 \pm 17.63)$ and interproximal surfaces (26.41 $\pm 22.77 v s .23 .12 \pm 20.98)(p>0.05)$. In conclusion, on Day 0, Group A presented higher efficacy in supragingival plaque removal than Group $B$, as reflected by whole-mouth, gumline and interproximal plaque scores.

Received: May 2020; Accepted: September 2020.

Keywords: toothbrushing - biofilms - oral hygiene

\section{Eficácia de duas escovas de cerdas macias na remoção de Placa. Um ensaio clínico randomizado}

\begin{abstract}
RESUMO
$O$ objetivo desse ensaio clínico, examinador-cego, randomizado e controle foi de comparadas a eficácia de duas escovas de cerdas macias em relação ao controle de placa. Setenta voluntários foram randomicamente alocados para Grupo A (escova com ponta conica) ou Grupo B (escova com ponta arredondada). Na consulta inicial (dia 0), voluntários receberam exame de placa utilizando o Índice de identificação de placa melhorado. Sob supervisão, eles escovaram seus dentes por 1 minuto com as escovas designadas e o exame de placa foi repetido. Voluntários continuaram seu regime de higiene oral (escova dental alocada e dentifrício comum fornecidos pelos pesquisadores) durante 7 dias. Os procedimentos da consulta inicial foram novamente repetidos. Análises estatísticas distintas foram realizadas para percentual de redução média de placa para os escores de boca toda, interproximal e linha gengival em ambos os momentos, utilizando teste de Mann-Whitney,
\end{abstract}

$p<0,05$. Após único uso da escova, no dia 0 , o percentual de redução média de placa foi significativamente reduzido em ambos os grupos $(p<0,05)$, com reduções significativas para boca toda $(21,39 \pm 12,44$ vs. 11,40 11,17$)$, linha gengival $(6,32 \pm 7,37$

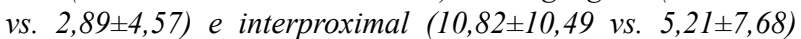
no Grupo A quando comparada com o Grupo B. Entretanto, no dia 7, nenhuma diferença significativa foi observada entre os grupos para boca toda $(29,94 \pm 20,91$ vs. 26,58 18,64$)$, linha gengival $(14,04 \pm 18,82$ vs. 13,78 $\pm 17,63)$ e interproximal $(26,41 \pm 22,77$ vs. $23,12 \pm 20,98)(p>0,05)$. Em conclusão, no dia 0 , a escova do Grupo A apresentou eficácia superior na remoção de placa suprageng ival quando comparada com a escova do Grupo B, como demonstrado nos escores de placa de boca toda, linha gengival e interproximal.

Palavras-chave: escovação dentária - bofilmes - higiene bucal 


\section{INTRODUCTION}

Supragingival plaque removal is undeniably the best way to ensure better oral health in the population. It has been proven to prevent caries, periodontal diseases and tooth loss and, more recently, to improve the outcome of dental implants ${ }^{1-3}$.

Oral hygiene routine demands time, dexterity and motivation. Therefore, the limits of the clinical effectiveness of self-performed oral hygiene are evident. Some studies have demonstrated the presence of remaining biofilm despite efforts to remove dental plaque ${ }^{2,4}$. The new classification of periodontal diseases and conditions established $10 \%$ as maximum number of bleeding sites for patients to be classified as healthy, which is achieved with good oral hygiene habits ${ }^{3,5}$.

Some studies have investigated the use of oral hygiene products, showing that there has been an increase worldwide. For example, while in Brazil in the 1990s, less than one toothbrush per capita was used per year ${ }^{4,6}$, this use had virtually doubled by $2010^{5,7}$. However, the estimation of use of oral hygiene products does not necessarily imply better clinical results in effectiveness of plaque control.

Toothbrushes are the gold standard product used to remove plaque from teeth. They differ in texture, tip, hardness, and even in their material, which may be natural or synthetic. The literature contains few studies comparing the efficacy of available toothbrushes. The use of a soft-bristle toothbrush has been recommended to improve plaque reduction while minimizing gingival tissue lesions ${ }^{8,9}$. Studies comparing the efficacy of available products are therefore important to better support the indication of any given toothbrush.

The aim of the present examiner-blind randomized controlled clinical study was to compare the efficacy two soft-bristle toothbrushes in terms of plaque removal with regular fluoride toothpaste in controlling established dental plaque over a 7-day period.

\section{MATERIAL AND METHODS}

\section{Study Design}

This study was designed as a phase III randomized, single-center, two-cell, examiner-blind, parallelgroup clinical study.

\section{Ethical Considerations}

The protocol was approved by the Institutional Review Board of the Federal University of Rio
Grande do Sul, Brazil and all the volunteers signed an informed consent form. The study was conducted according to good clinical practice standards ${ }^{10}$.

\section{Sample Size Estimate}

Sample size was estimated after a pilot study. Based on the standard deviation for the response measures of 0.58 , a significance level of $\alpha=0.05$ and $80 \%$ level of power, the present study was powered to detect a minimal statistically significant difference between study group means of $15 \%$, and 32 individuals per group were considered necessary. A $10 \%$ possible attrition rate was added. Thus, 35 individuals per group were considered for participation.

\section{Participants}

Seventy healthy male and female individuals, aged 21-70 years, were enrolled. Recruitment was performed at the Federal University of Rio Grande do Sul, Brazil. The following inclusion criteria were used: good oral health; initial mean plaque index of at least 0.6 , determined by the Improved Plaque Identification Index ${ }^{6,11}$; and $\geq 20$ natural uncrowned teeth, excluding third molars. Individuals were not included in the study if they had orthodontic bands, removable partial dentures, tumor or significant pathology in the soft or hard tissues of the oral cavity, moderate or advanced periodontal disease (purulent exudate, tooth mobility, and/or extensive loss of periodontal attachment or alveolar bone), antibiotic use in the month prior to entering the study, participation in any other clinical study, pregnant or breast-feeding status, dental prophylaxis in the 2 weeks prior to the baseline examination, history of allergy to oral/personal care consumer products or their ingredients, use of any prescription medicine that might interfere with the study outcome, medical condition preventing abstinence form eating/drinking/chewing gum for 4 hours prior to the scheduled visit, and history of alcohol or drug abuse.

\section{Experimental Procedures}

Participants were instructed to refrain from any oral hygiene measure for 12 hours before reporting to the clinical site, and from eating, drinking or smoking for 4 hours. The baseline examination comprised evaluation of the oral cavity soft tissue and perioral region followed by plaque disclosure with $10 \mathrm{ml}$ of $0.04 \%$ basic fuchsine solution (Replasul; Iodontosul, 
Porto Alegre - RS, Brazil). On Day 0, plaque examination was performed using the Improved Plaque Identification Index before toothbrushing ${ }^{11}$. Supragingival plaque was assessed on the facial and lingual surfaces of each tooth. Scores of 0 to 4 were assigned to all disclosed surfaces of the maxillary and mandibular teeth using a dental light and dental mirror. Teeth were divided into nine units (Fig. 1, A-I). From these site-wise scores, a whole-mouth plaque score was determined for each participant by calculating the proportion of sites in the mouth at which plaque was present. Three areas are near the gingival margin, two are interproximal, and four are on the body of the tooth. This index enables stratification of area(s) that are of concern during the analysis.

Simple randomization was performed by a computergenerated list by an external researcher responsible for allocation concealment. Toothbrushes were kept inside numbered opaque plastic bags. The experimental groups were:

Group A. A soft-bristle, tapered-tip toothbrush

Group B. A soft-bristle, end-rounded toothbrush.

All participants were instructed to brush their teeth for one minute under supervision with the assigned toothbrush and a commercially available standard fluoride toothpaste (Colgate Cavity Protection; Colgate, São Paulo, Brazil), after which plaque was evaluated (Day 0 after toothbrushing). They were instructed to use the product at home twice daily (morning and evening) for the next 7 days and to refrain from any interproximal cleaning. On day 7 , all participants returned to the clinical facility and the procedures performed on Day 0 were repeated. The same examiner (CKR), who was unaware of group allocation, performed all plaque examinations.

\section{Statistical Analysis}

The main study outcome is plaque score reduction, determined by the Improved Plaque Identification Index. Plaque Index was dichotomized into absence of dental plaque (scores 0 or 1 ) and presence of dental plaque (scores 2, 3 or 4). The percentage of sites with presence of dental plaque was calculated for each individual for both experimental periods (Day 0 and Day 7). The percentage reductions of plaque before and after toothbrushing were calculated for each experimental period as follows: percent of dental plaque after toothbrushing x 100 / percent of dental plaque before toothbrushing.

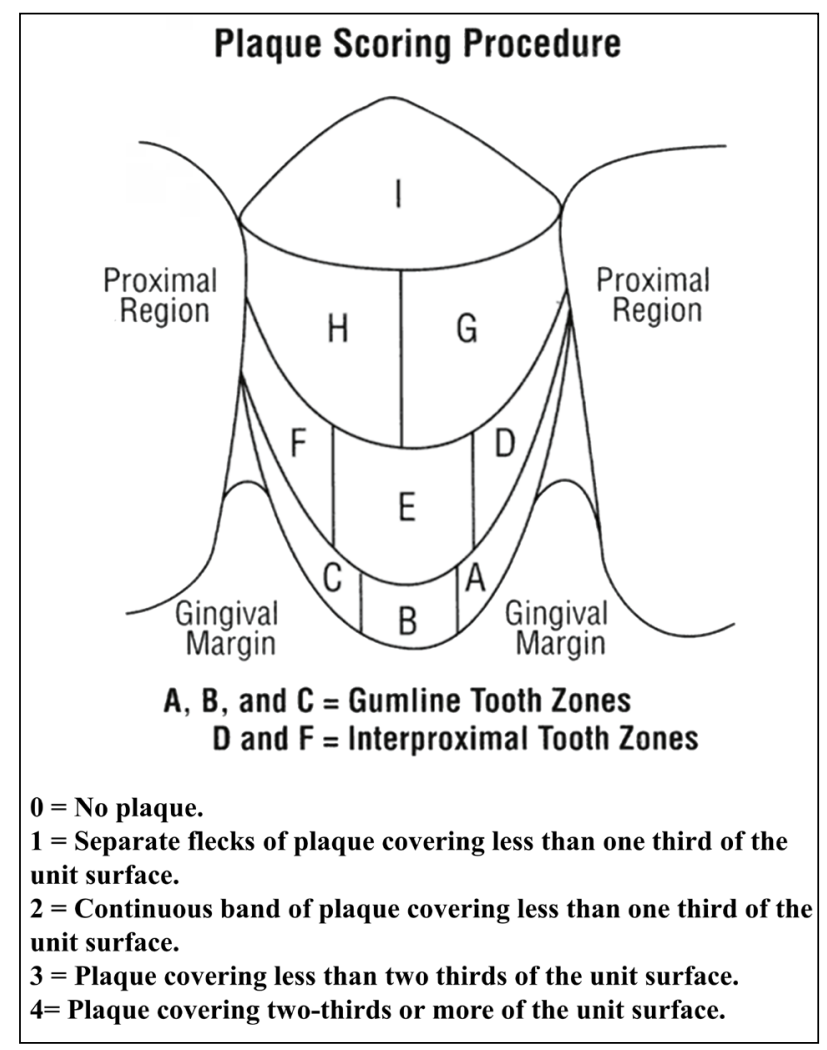

Fig. 1: Improved plaque identification index (according to Ayad et al., 2016).

Separate statistical analyses were performed for whole-mouth, interproximal, and gumline plaque scores. A per-protocol analytical approach was used. The statistician was blinded to participants' product allocation. Percentage reductions between before toothbrushing and after toothbrushing were calculated for both Day 0 and Day 7 for whole-mouth, interproximal and gumline regions. Comparisons between groups were performed using the Mann-Whitney test, as a non-parametrical data distribution was observed (Kolmogorov-Smirnov test showed a p-value $<0.05$ ).

Non-parametrical distribution was also identified for the number of present teeth, so the Mann-Whitney test was used to compare the number of teeth between groups. Age was compared between groups by t-test for independent samples. Categorical data (sex and smoking exposure) were compared between groups by chi-square test. A significance level of $\alpha=0.05$ was established for all analyses. Data analysis was performed using the statistical package SPSS 21.0 (SPSS Inc., Chicago, USA). 


\section{RESULTS}

One individual of the 71 initially screened for the study was not included (Fig.2). Out of the 70 included participants, 67 completed the 7-day clinical trial. Three participants -2 in Group A and 1 in Group B did not complete the study. Reasons for dropouts are mentioned in Fig. 2. Groups did not differ in gender or mean age (Table 1).

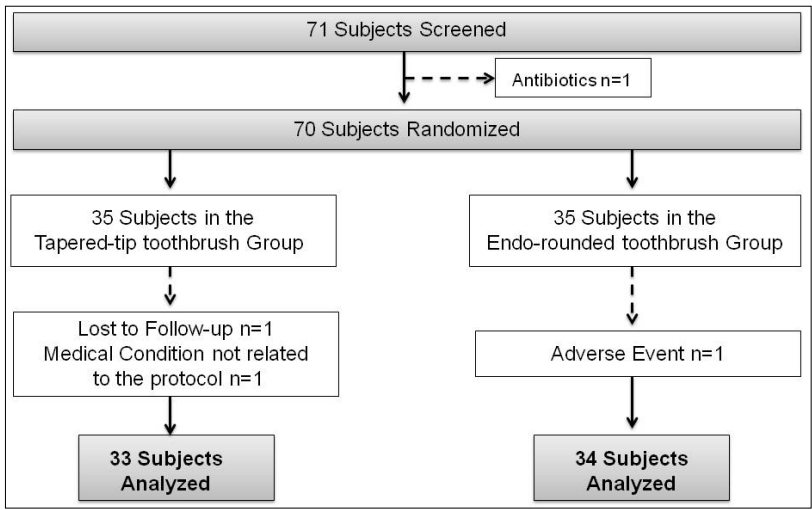

Fig. 2: Study Flowchart.

With relation to scores on Day 0 before toothbrushing, a statistically significant difference between groups was detected $(\mathrm{P}<0.001)$ (Table 1$)$, and therefore the present study analyzes percent reductions rather than means. Fig. 3 shows the mean percent reduction (from before toothbrushing to after toothbrushing) on Day 0, considering the wholemouth, interproximal and gumline and surfaces. Group A showed significantly greater reduction in mean whole-mouth $(21.39 \pm 12.44 \%)$, interproximal $(10.82 \pm 10.49 \%)$ and gumline $(6.32 \pm 7.37 \%)$ plaque scores compared to Group B, in which reductions were $11.4 \pm 11.17 \%, 5.21 \pm 7.68 \%$ and $2.89 \pm 4.57 \%$, respectively (Fig. 3).

In the Day 7 follow-up, a statistically significant difference was observed in the scores before

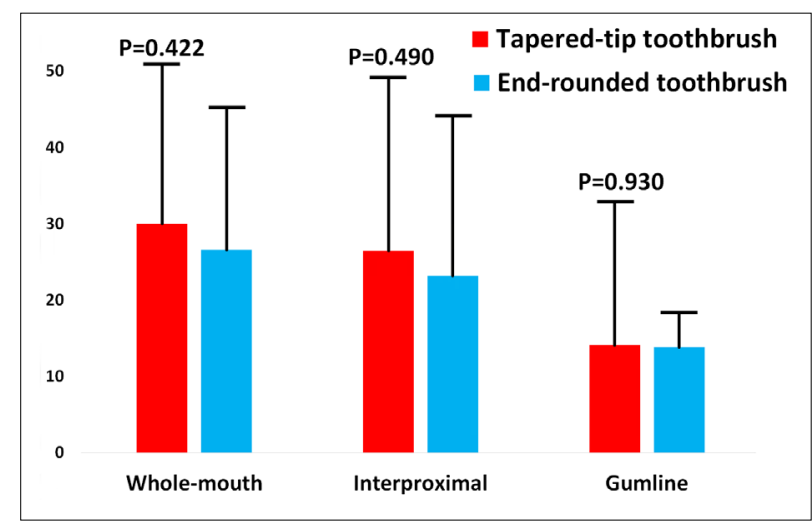

Fig. 3: Mean percent reduction of plaque for Group $A$ (tapered-tip toothbrush) and Group B (end-rounded toothbrush) on Day 0.

toothbrushing $(\mathrm{P}<0.007) \quad$ (Table 1) in wholemouth surfaces. Participants in Group A showed no significant difference in the whole-mouth (29.94 $\pm 20.91 \%)$, interproximal $(26.41 \pm 22.77 \%)$ or gumline $(14.04 \pm 18.82)$ percent reduction of plaque scores when compared to Group B, which had $26.58 \pm 18.64 \%, 23.12 \pm 20.98 \%$ and $13.78 \pm 17.63$, respectively. However, no statistically significant difference was observed in mean percent reduction of plaque (Fig. 4).

Three adverse events possibly related to the study were reported during the 7-day study: traumatic brushing, mouth ulcers and severe pain in teeth irradiating to ear and jaw as reported by the subjects. All three (3) adverse events were reported in Group $\mathrm{B}$ and one individual preferred not to continue in the study due to the discomfort. All participants who reported adverse events were carefully followed until the issue was resolved. As toothbrushing technique may explain these findings, all individuals received individualized oral hygiene instruction after all experimental periods were completed.

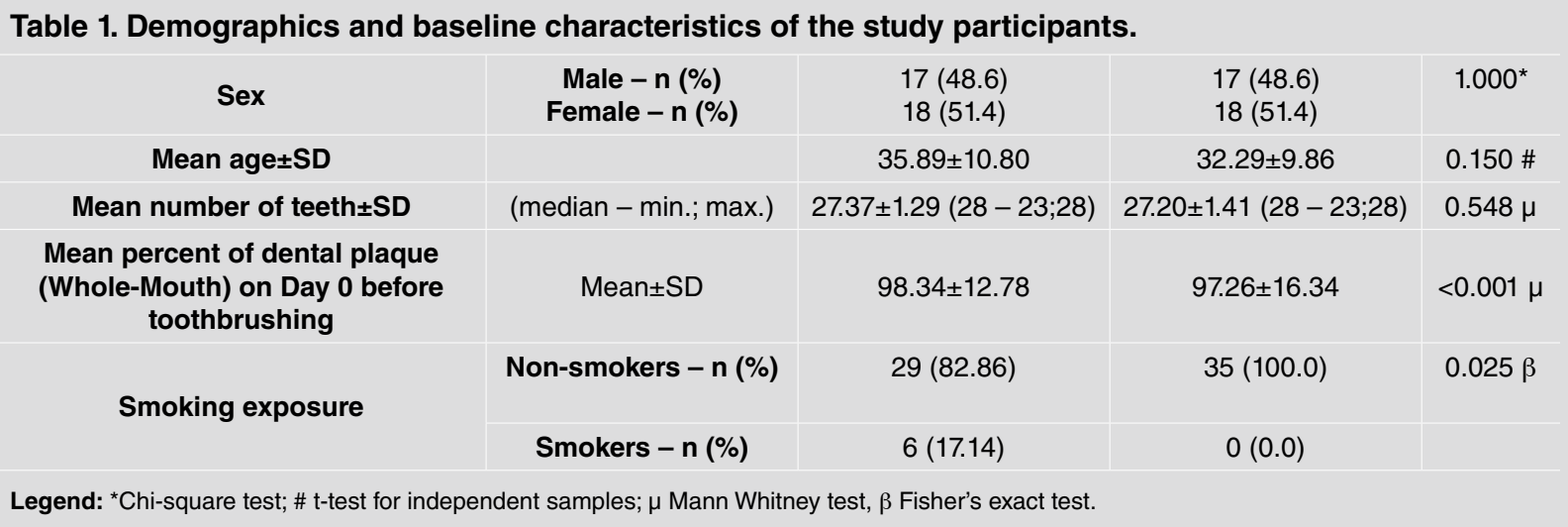

\begin{tabular}{|c|c|c|c|c|}
\hline Sex & $\begin{array}{c}\text { Male - n (\%) } \\
\text { Female - n (\%) }\end{array}$ & $\begin{array}{l}17(48.6) \\
18(51.4)\end{array}$ & $\begin{array}{l}17(48.6) \\
18(51.4)\end{array}$ & $1.000^{*}$ \\
\hline Mean age $\pm S D$ & & $35.89 \pm 10.80$ & $32.29 \pm 9.86$ & $0.150 \#$ \\
\hline Mean number of teeth \pm SD & (median - min.; max.) & $27.37 \pm 1.29(28-23 ; 28)$ & $27.20 \pm 1.41(28-23 ; 28)$ & $0.548 \mu$ \\
\hline $\begin{array}{c}\text { Mean percent of dental plaque } \\
\text { (Whole-Mouth) on Day } 0 \text { before } \\
\text { toothbrushing }\end{array}$ & Mean \pm SD & $98.34 \pm 12.78$ & $97.26 \pm 16.34$ & $<0.001 \mu$ \\
\hline \multirow{2}{*}{ Smoking exposure } & Non-smokers - $\mathrm{n}(\%)$ & $29(82.86)$ & $35(100.0)$ & $0.025 \beta$ \\
\hline & Smokers - n (\%) & $6(17.14)$ & $0(0.0)$ & \\
\hline
\end{tabular}




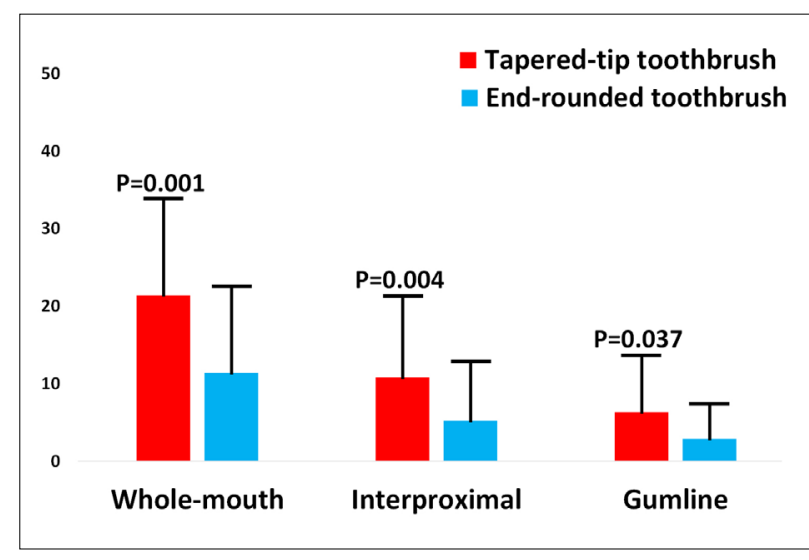

Fig. 4: Mean percent reduction of plaque for Group A (tapered-tip toothbrush) and Group B (end-rounded toothbrush) on day-7.

\section{DISCUSSION}

The present study was designed as a phase III randomized, single-center, two-cell, examinerblind, parallel-group clinical study, with the aim of comparing the plaque removal efficacy of two soft-bristle toothbrushes in 70 healthy volunteers. Plaque reduction was greater in Group A (taperedtip toothbrush) than in Group B (end-rounded toothbrush) in the first usage. This result was statistically significant in the percent reduction on Day 0 but was not significant on Day 7 .

Toothbrushing is known to be the most frequently used method of oral hygiene. The contemporary paradigm of oral hygiene is based on the highest efficacy with the least harm. In order to increase effectiveness and reduce the effects of improper brushing techniques, manufactures set the goal of evolving their toothbrushes in terms of design and bristle characteristics. It is well known that softbristle toothbrushes are efficacious and have less harmful potential ${ }^{8,9}$. Different toothbrushes have been developed with the aim of achieving better cleaning effect in the whole mouth, regardless of the type of movements used by the user ${ }^{12,13}$. A variety of studies can be found in the literature regarding different toothbrush designs and morphologies. It is not easy to compare the different studies, particularly due to high variety in design, follow-up, brands used, etc. The findings of such studies are important as different types of toothbrushes are launched on the market and there is constant need of support from sound studies in terms of efficacy and safety.

The present study was designed to compare the effect of a novel soft-bristle toothbrush (Group A) with a toothbrush that is already well established on the market (Group B). It should be emphasized that even though both brushes are considered soft, the brush being tested in the study (Group A) has softer bristles. Although it is well known that softer bristles can be less efficacious ${ }^{14}$, there is no doubt about the benefit of their being less harmful ${ }^{8}$. This is not a double-blind study, since the participants received different toothbrushes. However, all additional procedures were identical for both groups. The study was designed as an efficacy study, which demonstrates the potential of a novel device. Even though initial plaque scores were numerically similar, they differed significantly between groups, so in order to normalize data, the present study analyzes percent reduction in plaque scores. The results showed that Group A presented higher efficacy in reducing whole-mouth and interproximal plaque on Day 0 (which was the first contact with the brushes), even though the bristles were softer. The results after 7 days did not present a statistically significant difference. The separate analyses performed in his study are important because they consider the ability of the different types of toothbrushes to act on specific parts of the teeth. Their ability to remove plaque from interproximal areas, where other devices (such as dental floss) present limitations, is of special interest in terms of occurrence of dental caries, gingivitis and periodontitis.

Plaque was measured using the Improved Plaque Identification Index ${ }^{11}$, which was designed to quantify the presence of dental plaque in each of the 9 regions identified on teeth by the Navy Plaque Index. The Improved Plaque Identification Index could be more sensitive and provide more accurate demonstration of differences in plaque levels. However, this kind of quantification tends to generate regression to the mean, so we dichotomized the index to facilitate clinical interpretation. The results showed higher efficacy in Group A upon the first usage. The leveling of the results on Day 7 might be explained in part by the Hawthorne effect, since participant behavior may be expected to change in response to awareness of being observed ${ }^{15}$. In addition, the effect of a learning curve should not be disregarded.

Tapered bristle toothbrushes have been tested and shown to be more effective in reaching not only interproximal areas of the teeth along the gingival margin and under gumline, where plaque 
accumulates the most, but also to reach fissures, when compared to end-rounded bristles. This result was also positive in an in vitro study with artificial plaque in which tapered-tip bristles have demonstrated significantly better results in reducing plaque scores in the interproximal area ${ }^{16}$. The use of tapered-tip bristle toothbrushes could benefit patients who do not include flossing in their daily hygiene routine, as it was shown in an updated review $^{17}$ that flossing did not necessarily reduce dental caries and periodontal disease.

As in the study by Rösing et al. $2016^{18}$, a 12-hour period established to refrain from any type of oral hygiene before both evaluations showed that plaque accumulation was sufficient to test the efficacy of the toothbrushes, although it was significantly different before toothbrushing Day 0 in Group A. It should be noted that no brushing technique was imposed on the participants, in order to highlight the outcome of their real daily brushing techniques.

Three adverse events were reported in the present study, none of which was related to the study protocol. Most of the reported adverse events could be explained by causes related to patients' brushing habits. One of the participants was excluded. It is also important to highlight that soft and extra-soft bristle toothbrushes have been reported in a systematic review to be safer than other toothbrush stiffness when oral injuries are evaluated, with tapered or rounded bristle end-shape ${ }^{8}$ presenting no clinical relevance concerning soft oral tissue damage.

Participants were asked to brush their teeth for one (1) minute. Many studies have used the same brushing time, the main reason being to avoid the

\footnotetext{
ACKNOWLEDGEMENT

This study was sponsored by Colgate Palmolive Company. Zilson Malheiros and Bernal Stewart are currently employed by Colgate Palmolive Company. The other authors are independent researchers.
}

\section{FUNDING}

This study was financed in part by the Coordenação de Aperfeiçoamento de Pessoal de Nível Superior - Brasil (CAPES) - Finance Code 001. patient needing to brush excessively ${ }^{18-20}$. Moreover, it has been demonstrated that patients do not spend longer than one minute on toothbrushing in general. This study has strengths and limitations that should be noted. Among the strengths are the study design, with randomization of the participants, use of a standardized brushing time, examiner reproducibility, examiner and statistician blinding, and very high compliance rate. The limitation of the study is the efficacy design, in which a short time ( 7 days) was used. Neither effectiveness nor effect on gingival inflammation can be ascertained from our results.

Despite the abovementioned limitations, it should be noted that there were better interproximal results in Group A, which may be relevant, considering that toothbrushes were not meant to reach this area. Other studies have demonstrated similar results with tapered-tip toothbrushes ${ }^{18-20}$. It is important to remember that a systematic review published by the Cochrane collaboration and revisited in 2019 showed concerning results regarding the levels of periodontal disease and caries in dental floss users, concluding that dental floss users do not necessarily have less diseases ${ }^{17}$. Thus, it is always important to study newly designed toothbrushes, especially as a possibility of compensating for the limited effects of flossing, according to how they are used. Longer term randomized controlled trials are warranted, including the analysis of adverse events, in order to prevent gingival tissue harm.

The tapered-tip toothbrush performed better than the end-rounded toothbrush in terms of efficacy at the first evaluation.

\section{CORRESPONDENCE}

Dr. Cassiano Kuchenbecker Rösing

Federal University of Rio Grande do Sul

Av. Ramiro Barcelos, 2492

90035-003 - Porto Alegre - RS

Brazil

ckrosing@hotmail.com 


\section{REFERENCES}

1. Axelsson P, Nystrom B, Lindhe J. The long-term effect of a plaque control program on tooth mortality, caries and periodontal disease in adults. Results after 30 years of maintenance. J Clin Periodontol 2004;31:749-757.

2. Jepsen S, Berglundh T, Genco R, Aass AM et al. Primary prevention of peri-implantitis: managing peri-implant mucositis. J Clin Periodontol 2015;42 Suppl 16:S152-157.

3. Lindhe J, Nyman S. Long-term maintenance of patients treated for advanced periodontal disease. J Clin Periodontol 1984;11:504-514.

4. Halla-Junior R, Oppermann RV. Evaluation of dental flossing on a group of second grade students undertaking supervised tooth brushing. Oral Health Prev Dent 2004;2:111-118.

5. Chapple ILC, Mealey BL, Van Dyke TE, Bartold PM et al. Periodontal health and gingival diseases and conditions on an intact and a reduced periodontium: Consensus report of workgroup 1 of the 2017 World Workshop on the Classification of Periodontal and Peri-Implant Diseases and Conditions. J Periodontol 2018;89 Suppl 1:S74-s84.

6. Gjermo P, Rosing CK, Susin C, Oppermann R. Periodontal diseases in Central and South America. Periodontol 2000 2002;29:70-78.

7. Oppermann RV, Haas AN, Rosing CK, Susin C. Epidemiology of periodontal diseases in adults from Latin America. Periodontol 2000 2015;67:13-33.

8. Ranzan N, Muniz F, Rösing CK. Are bristle stiffness and bristle end-shape related to adverse effects on soft tissues during toothbrushing? A systematic review. Int Dent J 2019;69:171182.

9. Hoogteijling F, Hennequin-Hoenderdos NL, Van der Weijden GA, Slot DE. The effect of tapered toothbrush filaments compared to end-rounded filaments on dental plaque, gingivitis and gingival abrasion: a systematic review and meta-analysis. Int J Dent Hyg 2018;16:3-12.

10. Grimes DA, Hubacher D, Nanda K, Schulz KF, Moher D, Altman DG. The Good Clinical Practice guideline: a bronze standard for clinical research. Lancet (London, England) 2005;366:172-174.
11. Ayad FN, Salim A, Elias A, Stewart B, Panakagos F. Improved plaque identification index. United States Patent Application Publication US. USA; 2016.

12. Terrana A, Rinchuse D, Zullo T, Marrone M. Comparing the plaque-removal ability of a triple-headed toothbrush versus a conventional manual toothbrush in adolescents with fixed orthodontic appliances: A single-center, randomized controlled clinical trial. Int Orthod 2019;17:719-725.

13. Xu Z, Cheng X, Conde E, Zou Y, Grender J, CcahuanaVasquez RA. Clinical assessment of a manual toothbrush with CrissCross and tapered bristle technology on gingivitis and plaque reduction. Am J Dent 2019;32:107-112.

14. Zanatta FB, Bergoli AD, Werle SB, Antoniazzi RP. Biofilm removal and gingival abrasion with medium and soft toothbrushes. Oral Health Prev Dent 2011;9:177-183.

15. Berthelot JM, Le Goff B, Maugars Y. The Hawthorne effect: stronger than the placebo effect? Joint Bone Spine 2011; 78:335-336.

16. Hotta M, Imade S, Kotake H, Sano A, Yamamoto K. Artificial plaque removal from interproximal tooth surfaces (maxillary premolar and molar) of a jaw model. Oral Health Prev Dent 2009;7:283-287.

17. Sambunjak D, Nickerson JW, Poklepovic Pericic T, Johnson TM et al. WITHDRAWN: Flossing for the management of periodontal diseases and dental caries in adults. Cochrane Database Syst Rev 2019;4:CD008829.

18. Rosing CK, Cavagni J, Gaio EJ, Muniz FW et al. Efficacy of two soft-bristle toothbrushes in plaque removal: a randomized controlled trial. Braz Oral Res 2016;30:e134.

19. Gallob J, Petrone DM, Mateo LR, Chaknis P et al. Comparative Efficacy of a Soft Toothbrush with Tapered-tip Bristles and an ADA Reference Toothbrush on Established Gingivitis and Supragingival Plaque over a 12-Week Period. J Clin Dent 2016;27:39-47.

20. Sowinski J, Petrone DM, Wachs GN, Chaknis P et al. Efficacy of three toothbrushes on established gingivitis and plaque. Am J Dent 2008;21:339-345. 